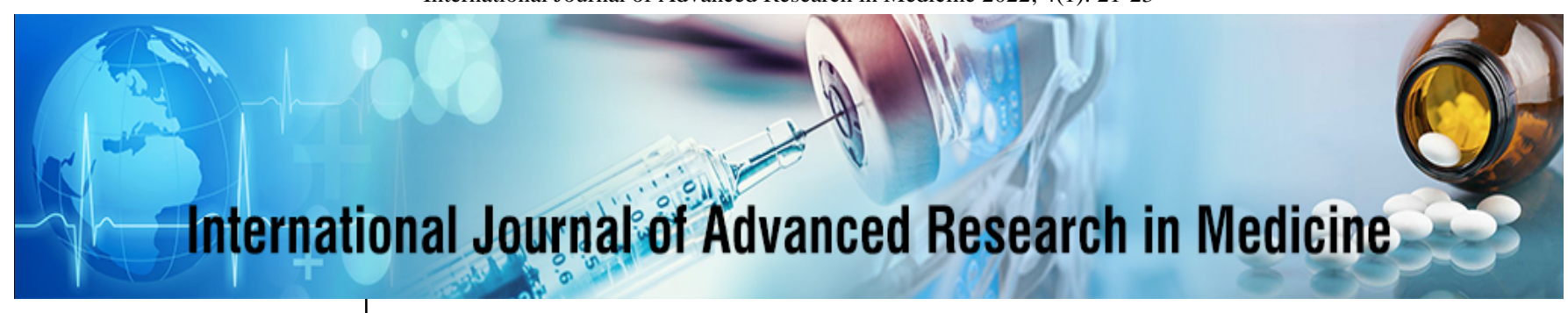

E-ISSN: 2706-9575

P-ISSN: 2706-9567

IJARM 2022; 4(1): 21-23

Received: 05-10-2021

Accepted: 16-12-2021

Nameirakpam Devakanta Singh

Associate Professor,

Department of ENT, JNIMS,

Imphal, Manipur, India

Yumnam Tomba Singh

Associate Professor,

Department of ENT, JNIMS,

Imphal, Manipur, India

Takhellambam Biram Singh Assistant Professor,

Department of ENT, JNIMS,

Imphal, Manipur, India
Corresponding Author:

Takhellambam Biram Singh

Assistant Professor,

Department of ENT, JNIMS,

Imphal, Manipur, India

\section{Incidence of allergic conjunctivitis in patients with allergic rhinitis: A randomized observational study}

\author{
Nameirakpam Devakanta Singh, Yumnam Tomba Singh and \\ Takhellambam Biram Singh
}

DOI: https://doi.org/10.22271/27069567.2022.v4.i1a.315

\begin{abstract}
Background: Although nasal allergy has been prominent in allergy research, ocular allergy is increasingly recognized as a distinct symptom complex that imposes its own disease burden and reduction in patients' quality of life. In the past year, knowledge of the relationships between allergic conjunctivitis (AC) and allergic rhinitis (AR) has increased. AC is commonly manifesting as itchy or watering or red eye, comprising the symptoms of the total ocular symptom scores (TOSS). Allergic conjunctivitis is highly prevalent and has a close epidemiologic relationship with allergic rhinitis. Both conditions also exhibit similar pathophysiologic mechanisms. Therefore, the objective of the present study was to identify the incidence of AC in patients with AR.

Methods: This randomized observational study was conducted on 150 patients for a 6 month period from June to November 2021, having diagnosed AR and attending the outpatient clinic of our department. The patients were directly questioned if they had AC, clarified by using standard screening questions of red, itchy and watery eyes and quantified by TOSS and were asked about indirect symptoms that may be attributable to AC.

Results: Among the 150 patients, 54.66\% of patients identified AC on direct questioning; additional symptoms were squint at $41.33 \%$ and blinking at $52.66 \%$. Olopatadine, significantly reduced TOSS scores within 5 minutes of treatment and $82.66 \%$ showed improvement, which identified $44.66 \%$ silent sufferers of AC. A total of $94 \%$ AC subjects were identified through TOSS symptoms and totally 96.66\%, detecting additional symptoms.

Conclusions: The screening questions could identify only about $54.66 \%$ of the patients with AC. Additional specific questioning and a therapeutic challenge in suspected patients can help identify patients who may benefit from treatment of AC.
\end{abstract}

Keywords: Allergic conjunctivitis, allergic rhinitis, olopatadine

\section{Introduction}

Allergic rhinitis (AR) is a chronic inflammatory disorder of the nasal mucosa caused by IgEmediated early- and late-phase hypersensitivity responses ${ }^{[1]}$. AR symptoms include rhinorrhea, nasal obstruction and blockage, nasal itching, and repetitive sneezing. It is also often accompanied by allergic conjunctivitis (AC) with symptoms that can include itchy, red, watery, and/or swollen eyes ${ }^{[2]}$.

Because of the non-life-threatening nature of symptoms, AR and AC have, in the past, been considered trivial diseases but are increasingly recognized as having a major effect on quality of life (QOL), emotional well-being, sleep, daily activities, and productivity when poorly controlled ${ }^{[3]}$.

According to the classification of ocular allergy proposed in 2006 by the International Ocular Inflammation Society (IOIS), based on immunopathological mechanisms, allergic conjunctivitis (AC) is a type of ocular allergy which in turn can be subdivided into seasonal allergic conjunctivitis (SAC) and perennial allergic conjunctivitis (PAC) ${ }^{[4]}$.

Under-recognition of $\mathrm{AR}$ is common, with the proportion of undiagnosed AR patients ranging from 25-60\%. Clinically, AC patients have heightened sensitivity and rubbing of eyelids can contribute to dermatitis, making patients focusing more on the dermatitis than conjunctival symptoms ${ }^{[5]}$.

Therefore, this study took place among pediatrics and adults within a rural population. The prime objective of the present study was to identify the incidence of AC in patients with AR, which were quite reliable to ascertain AC severity and we aimed to investigate the interrelationship between the two conditions, further. 


\section{Methods}

A randomized observational study was conducted on 150 patients both children and adults, for a 6 month period from February to July 2019, which included the pollen season, having diagnosed AR and attending the outpatient clinic in our department. The patients were directly questioned if they had AC, clarified by using standard screening questions of red, itchy and watery eyes and quantified by TOSS, scored as a percentage from 0 to 100 . Patients were asked about indirect symptoms that may be attributable to AC: eyelid dermatitis, frequent blinking, eye sensitivity and frontal headache.

Patients were prospectively diagnosed with AR based on clinical history, examination and skin prick testing by an allergy specialist. They were given a drop of olopatadine in each eye to help identify silent disease. Olopatadine hydrochloride $0.1 \%$ was selected for its efficacy in AC, providing negligible side effect profile and rapid onset of action, evident from five minutes post administration ${ }^{[6,7]}$. Fifty healthy controls, without a clinical history of AR or AC or SAC were also treated with olopatadine drops to determine if there was a non-specific lubricating effect of olopatadine hydrochloride and included in the study.

Exclusion criteria: Patients on antihistamines or refusing to reply to questionnaire or having history of other kinds of allergies or not consenting to participate in the study were excluded.

Ethical approval was obtained from institutional ethics committee. Data was analyzed using Microsoft excel 2010 software using a paired $\mathrm{t}$ test. A Pearson correlation coefficient was used to compare relationship between TOSS positivity and presence of additional symptoms.

\section{Results}

Among the 150 patients, 85 were females and 65 males, with average age $34 \pm 6.83$ years old. 82 (54.66\%) of patients identified themselves as having AC on direct questioning and upon enquiring about specific TOSS symptoms. Additionally, symptoms shown by patients like squint 62 (41.33\%), blinking 79 (52.66\%), eyelid dermatitis 68 (45.33\%) and frontal headache 69 (46\%) were possible symptoms attributable to AC. Administration of antihistamine, olopatadine, significantly reduced TOSS scores within 5 minutes of treatment. 124 (82.66\%) subjects showed improvement as against $26(17.33 \%)$ of those who showed no change in ocular symptoms. Based on a negative history of AC and baseline TOSS of 0 , therapeutic challenge of olopatadine identified 67 (44.66\%) silent sufferers of AC, whereas no effect on TOSS was observed in 30 healthy controls treated with olopatadine. 99 (90\%) AC subjects were identified through TOSS symptoms. Plausible indirect AC symptoms detected 141 (94\%) subjects. However, combining standard TOSS and additional questions detected 145 (96.66\%) AC subjects.

\section{Discussion}

Interest in allergic conjunctivitis (AC), isolated or associated to allergic rhinitis (AR), has increased in recent years due to its high and growing prevalence, the important healthcare costs generated by the disease, and its impact upon patient quality of life. Previous studies have linked Allergic Rhinitis (AR) to be co-existed with another form of allergic disorders including Allergic Conjunctivitis (AC), Atopic dermatitis and Allergic Asthma ${ }^{[8,9]}$. Most of the study showed the prevalence of AC was $30 \%$ to $40 \%$ to be associated with rhinitis, while $30 \%$ had eczema and $24 \%$ had asthma ${ }^{[10,11]}$. In our study AC was identified in 54.66\% of patients with AR using direct questioning in relation to history of AC.

On 50 healthy, non-atopic controls, olopatadine therapeutic challenge was performed to ensure against the nonspecific lubricating effect of the eye drop as a lubricant could have improved symptoms. There was no specific improvement in controls that were given this lubricant effect and hence TOSS improvement can be easily attributed to olopatadine. The co-existence of AC was well recognized in patients with AR although coreporting frequency may be as low as $40 \%{ }^{\text {[12] }}$

Specific questioning regarding indirect symptoms increased the incidence of AC in patients with AR to $96.66 \%$. Under recognition of allergic conjunctivitis may be due to patients and physicians paying more attention to allergic comorbidities such as AR or rhinitis ${ }^{[13]}$.

Limitations of this study include; it was an open clinical audit and direct survey questions were used. Questions of children were occasionally influenced by their parent's answers or prompting.

\section{Conclusion}

This research was conducted to correlate the features of allergic rhinitis with those of conjunctivitis. The standard AC screening questions identified just over half of the patients with AC. As suggested by others, the absence of a history does not negate the value of examining the conjunctiva. Additionally we suggest that symptoms of blinking, squinting, eyelid dermatitis and frontal headache and use of olopatadine hydrochloride eye drops can help identify patients with "silent” symptoms.

\section{References}

1. Scadding GK. Optimal management of allergic rhinitis. Arch Dis Child. 2015;100:576-582.

2. Broz Ek JL, Bousquet J, Agache I, et al. Allergic Rhinitis and its Impact on Asthma (ARIA) guidelines 2016 revision. J Allergy Clin Immunol. 2017;140:950958.

3. Linneberg A, Dam Petersen K, Hahn-Pedersen J, Hammerby E, Serup-Hansen N, Boxall N. Burden of allergic respiratory disease: a systematic review. Clin Mol Allergy. 2016;14:12.

4. Leonardi A, De Dominicis C, Motterle L. Immunopathogenesis of ocular allergy: a schematic approach to different clinical entities. Curr Opin Allergy Clin Immunol. 2007;7(5):429-35.

5. Wüthrich B, Brignoli R, Canevascini M, Gerber M. Epidemiological survey in hay fever patients: symptom prevalence and severity and influence on patient management. Schweiz Med Wochenschr. 1998;128(5):139-43.

6. Bauchau V, Durham SR. Prevalence and rate of diagnosis of allergic rhinitis in Europe. ER J. 2004;24(5):758-64.

7. Kockaya G, Wertheimer A. Cost effectiveness analysis of five different treatment alternatives in seasonal allergic conjunctivitis. JAPS. 2011;1(5):72-5.

8. Perkin MR, Bader T, Rudnicka AR, Strachan DP, Owen CG. Inter Relationship between Rhinitis and Conjunctivitis in allergic Rhinoconjunctivitis and 
Associated Risk factors in Rural UK Children, PLOs. 2015. Available:

https://doi.org/10.1371/journal.pone.0143651Accessed 5th November 2018.

9. Singh K, Axelrod S, Bielory L. The epidemiology of ocular and nasal allergy in the United States, 1988 1994. J Allergy Clin Immunol. 2010;126:778-783.

10. Gradman J, Wolthers OD. Allergic conjunctivitis in children with asthma, rhinitis and eczema in a secondary outpatient clinic. Pediatr Allergy Immunol. 2006;17(7):524-6.

11. Nathan RA, Meltzer EO, Selner JC, Storms W. Prevalence of allergic rhinitis in the United States. J Allergy ClinImmunol. 1997;99(6):808-14.

12. Bousquet J, Cauwenberge PV, Khaltaev N. Allergic rhinitis and its impact on asthma. J Allergy Clin Immunol. 2001;108(5):147-334.

13. Neilson R, Bielory L. Epidemiology of allergic conjunctivitis. Curr Opin Allergy Clin Immunol. 2011;11(5):471-6. 